\title{
Bone remodeling in additive manufactured porous implants changes the stress distribution
}

Cheong, Vee San, Mumith, Aadil, Coathup, Melanie, Blunn, Gordon, Fromme, Paul

Vee San Cheong, Aadil Mumith, Melanie J. Coathup, Gordon W. Blunn, Paul Fromme, "Bone remodeling in additive manufactured porous implants changes the stress distribution," Proc. SPIE 11381, Health Monitoring of Structural and Biological Systems XIV, 113812V (22 April 2020); doi: $10.1117 / 12.2558093$

SPIE. Event: SPIE Smart Structures + Nondestructive Evaluation, 2020, Online Only 


\title{
Bone remodeling in additive manufactured porous implants changes the stress distribution
}

\author{
Vee San Cheong a,b), Aadil Mumith ${ }^{\text {a) }}$, Melanie J. Coathup ${ }^{\text {c) }}$, Gordon W. Blunn ${ }^{\text {a,d) }}$, Paul Fromme ${ }^{\text {b) }}$ \\ a) Institute of Orthopaedics and Musculoskeletal Science, University College London, Royal \\ National Orthopaedics Hospital, UK \\ b) Department of Mechanical Engineering, University College London, UK \\ c) College of Medicine, University of Central Florida, USA \\ d) School of Pharmacy and Biomedical Sciences, University of Portsmouth, UK
}

\begin{abstract}
Safety and efficacy of additive manufactured porous implants is a growing concern due to several, recent recalls. The safety of bone implants depends on the effects of implantation and partial bone ingrowth on stress and strain. Finite element analysis, using two new algorithms to simulate bone ingrowth, was verified against histology results for an ovine condylar critical sized defect model. Implants were manufactured from Ti6Al4V using selective laser sintering. Results showed that partial bone formation reduces stress concentrations to safe levels, improving the long-term fatigue resistance. Higher bone ingrowth was predicted for implants made from lower modulus Titanium-tantalum alloy.
\end{abstract}

Keywords: implant failure, stress shielding, laser sintered titanium alloy implant, bone ingrowth

\section{INTRODUCTION}

Achieving osseointegration is one of the main aims in Orthopedic implant design, as clinical and histology results from retrievals have demonstrated that biological and mechanical fixation improves the stability and survivorship of the implant [1-3]. Most commercially available implants are manufactured using bulk structural material, with a bioactive coating or porous coating, in the form of sintered beads or fiber meshes, applied on the dense substrate to promote bone ingrowth. However, these implants could still fail due to aseptic loosening, as the structural stiffness of bulk metallic implants are 5-20 times higher than that of bone [4]. Recent advancements in additive manufacturing have allowed for the fabrication of substantially porous implants that have effective elastic moduli matching that of bone, by altering the design and porosity $[1,5]$. Moreover, porous implants have an interconnected structure, which allows bone tissue to grow deeper into the structure [6]. Porous segmental prostheses manufactured by selective laser sintering have been demonstrated to promote increased osseointegration compared to machined implants with a grooved collar design in an in vivo sheep study [7].

The mechanical benefit of bone formation in additively manufactured implants was demonstrated recently by an experiment that showed that the mechanical properties of porous implants improve by a factor of 2-7 when completely and homogenously filled with epoxy. However, several in vivo studies have shown that bone formation in implants is typically incomplete [8-10]. The performance of porous implants fabricated by additive manufacturing is a concern, as literature has reported a higher than expected failure rate for a porous acetabular component, and a Class 2 device recall of posteriorlateral lumbar cages due to preoperative and postoperative fracture [11, 12]. Standardized mechanical testing for evaluating the fatigue performance of implants cannot simulate bone formation into the porous implants, and neither can in vivo testing evaluate implant safety effectively as the duration of the experiments is relatively short compared to the anticipated implant longevity $[6,13]$. The modelling of bone formation has been demonstrated to be able to predict extracortical bone formation into a grooved collar of the segmental prosthesis [14]. Hence, modelling partial and inhomogeneous bone ingrowth into porous implants can be used to assess the changes in stress distribution and relate findings to the yield and fatigue strength of the implant [15]. Moreover, it can be used to assess different implant designs and materials that improve clinical outcomes [4].

The aim of this study was to predict the extent of bone formation in two porous Ti6Al4V implants, and assess the changes to the stress distribution in the implant. This contribution analyses the results from an experimental dataset that compared the effects of different pore sizes and coating on the extent of bone ingrowth and osseointegration in an in vivo sheep model

Health Monitoring of Structural and Biological Systems XIV, edited by Paul Fromme,

Zhongqing Su, Proc. of SPIE Vol. 11381, 113812V · (c) 2020 SPIE

CCC code: $0277-786 \mathrm{X} / 20 / \$ 21 \cdot$ doi: $10.1117 / 12.2558093$

Proc. of SPIE Vol. 11381 113812V-1 
[9]. FEA models were developed to model the mechanical environment, and a mechanoregulation algorithm was applied to predict adventitious bone formation $[4,6,14]$. The numerical results were verified by comparing the extent of osseointegration, in the form of bone-to-implant contact (BIC), against histology results, before assessing the stress changes due to inhomogeneous bone formation in the implant. The material stiffness (Young's modulus) of the implant was also lowered, to assess if titanium tantalum alloys (TiTa), which can be laser sintered but are currently not in use in commercially available implants, can improve the implant performance and safety.

\section{MATERIALS AND METHODS}

\subsection{Implant design and animal study}

Cylindrical implants were selectively laser sintered (SLS) using Ti6Al4V with $14.5 \mathrm{~mm}$ length and $8 \mathrm{~mm}$ diameter (Fig. 1) as part of a larger study [9]. Two porous designs with pore sizes of $700 \mu \mathrm{m}$ and $1500 \mu \mathrm{m}$ were incorporated into the same implant, giving porosities of $75 \%$ and $53 \%$ respectively. The struts in the $700 \mu \mathrm{m}$ and $1500 \mu \mathrm{m}$ implants were designed with $300 \mu \mathrm{m}$ diameter, $700 \mu \mathrm{m}$ height, and $750 \mu \mathrm{m}$ diameter, $1500 \mu \mathrm{m}$ height, respectively. Critical size defects of $8 \mathrm{~mm} \times 15 \mathrm{~mm}$ were created in the medial condyles of the right and left hind limbs of mature sheep. The implants were press-fitted into the holes, before the periosteum, fascia and subcutaneous tissues were repaired. All animals were allowed to recover with unrestricted weight bearing, and no adverse effects due to surgery was observed. All procedures were carried out in accordance with the UK Animals (Scientific Procedures) Act 1986, under UK Government Home Office personal and project licenses, and approved by the Royal Veterinary College Ethical Review Committee.

The implants were retrieved for histological and histomorphometric analysis after 6 weeks; only one time period was used to assess the initial bone ingrowth and osseointegration of the implants, to reduce the number of animals used in experiments. The retrieved implants were trimmed of excess soft tissue and fixed in 10\% neutral buffered formalin (NBF). Fixed tissues were dehydrated, defatted and embedded in hard grade acrylic resin (LR White Resin, London Resin Company, UK). The resin blocks were sectioned in the longitudinal plane to obtain $80 \mu \mathrm{m}$ sections. The sections were stained with Toluidine Blue and Paragon to detect soft tissue and bone, respectively, and to evaluate the extent of bone ingrowth. This was assessed by measuring the bone-to-implant contact (BIC), which is the ratio of the length of bone in direct contact with the implant, to the total implant perimeter within the pores. The stained slides were imaged using light microscopy (Axioskop, Carl Zeiss, UK) while histomorphometry was conducted in ImageJ (v1.51, NIH, USA). MannWhitney U tests were conducted in Origin 2016 (OriginLab Corp., USA) to compare the BIC.

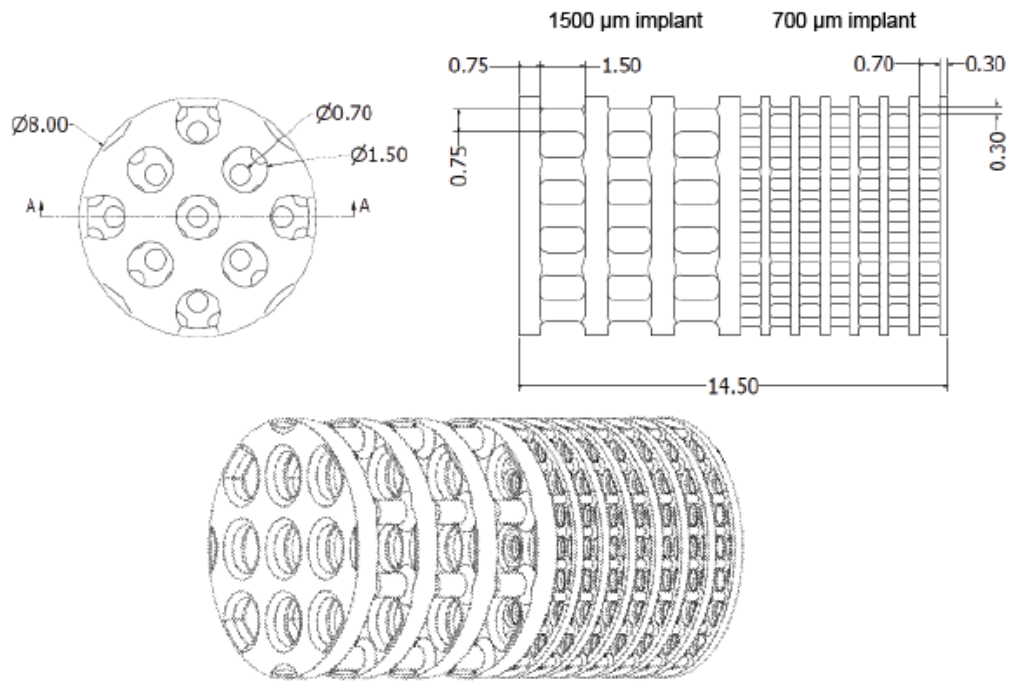

Fig. 1: Implant design with porosities of $75 \%$ and $53 \%$ for the $1500 \mu \mathrm{m}$ implant and $700 \mu \mathrm{m}$ implant respectively (units: mm). 
A

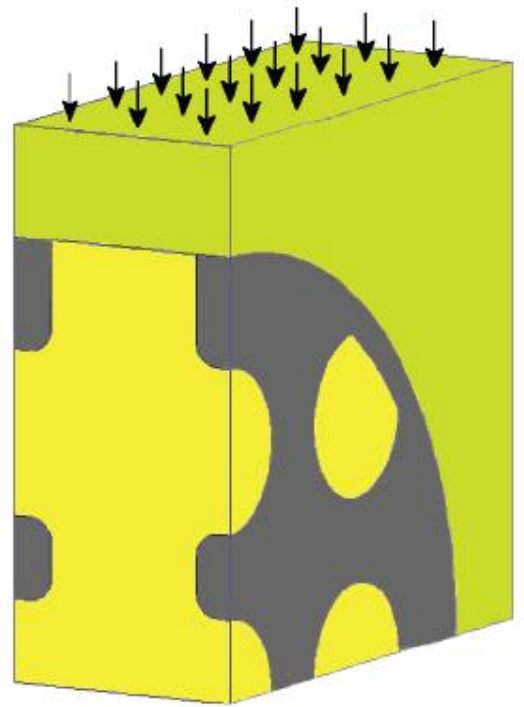

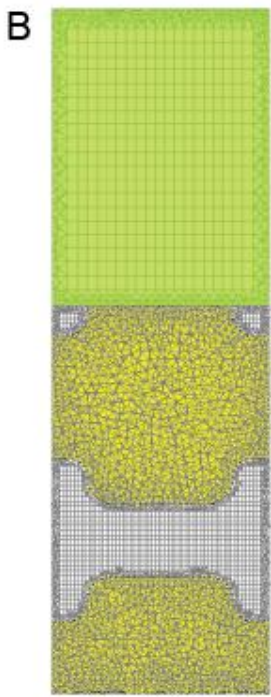

Fig. 2: (A) Boundary conditions and (B) mesh of the FEA model for $1500 \mu \mathrm{m}$ implant.

\subsection{Finite element model}

The implants were modelled separately as a quarter of a slice to reduce computational costs, as the implant orientation and location were not found to have an effect on the extent of bone ingrowth $[4,9]$. The implants were assumed to be surrounded by homogenized trabecular bone, and filled initially with soft tissue which was allowed to adapt its elastic modulus based on the local stimulus present (Fig. 2). Isotropic linear elastic properties were used for all material properties (Trabecular bone: Elastic modulus E $=1.5 \mathrm{GPa}$, Poisson's ratio $v=0.34$; Ti6Al4V: $110 \mathrm{GPa}, v=0.34$; Soft tissue: $0.5 \mathrm{GPa}, v=0.3$ ). The models were axially restrained and the peak axial load through the medial condyles of the stifle joint under walking gait was applied [16], scaled to the implant repeating pattern (700 $\mu \mathrm{m}$ implant: $89 \mathrm{~N} ; 1500 \mu \mathrm{m}$ implant: $200 N)$. The application of a peak load in a static analysis has been demonstrated to capture the main changes in bone remodeling [17].

The models were meshed with linear tetrahedral elements for the soft tissue and linear hybrid 4-, 6- and 8-noded elements for bone and implant to improve geometrical conformance while minimizing the model size. Higher-order elements were not used as the load transmitted through the condyles is primarily compression [6]. Micromotion was assumed to be negligible as the bone-implant interface is perpendicular to the direction of loading, thus tied contact conditions were used throughout. Static stress analysis was conducted for the mesh convergence study, with error criteria of 5\% and $1 \%$ at the fillet, and in the bone/soft tissue, respectively. The FEA models converged with mesh sizes of $0.06 \mathrm{~mm}$ and $0.03 \mathrm{~mm}$ for $1500 \mu \mathrm{m}$ and $700 \mu \mathrm{m}$ implants, respectively. MSC.Marc 2017.0 (MSC Software Corporation, USA) was used to mesh and solve the FEA models. The output of the FEA was the local strain energy density (SED), which was used to predict the extent of bone ingrowth and remodeling. A parametric study was conducted by varying the material properties of the implants to investigate the effect of the structural stiffness on the extent of bone remodeling, stress distribution, and safety of implants. A total of 4 material properties were evaluated as part of a larger study [4], but only the results for TiTa $(\mathrm{E}=67 \mathrm{GPa}, v=0.34)[18]$ is reported in this study.

\subsection{Bone adaptation algorithm}

A strain energy density (SED) based adaptive feedback algorithm was used for the simulation of bone remodeling into the pores of the implants [14]. Based on the mechanostat theory that bone responds to changes in mechanical stimulus by adding or removing bone [19], a trilinear curve was used, where high and low localized strain energy density led to increases and decreases in tissue density, and a 'lazy zone' where bone formation and resorption are balanced such that there is no change in the tissue density:

$$
(1-\delta) k \leq S E D \leq(1+\delta) k
$$


To model the physiological observation that bone grows from the edge of the implant towards the center of the porous structure [9], and no bone formation occurred independently in the middle of the implant, an algorithm termed connectivity was added to enforce the sequential laying down of new bone. This was achieved by permitting only elements adjacent to bone or an element that has begun remodeling, and with SED above the threshold, to adapt their densities:

$$
\frac{d \rho}{d t}=B(S E D-k) \quad 0<\rho \leq \rho_{c b}
$$

where $B$ and $k$ are the remodeling rate and reference threshold, set at values of $1\left(\mathrm{gcm}^{-3}\right)^{2} / \mathrm{MPa}$-ctu and $0.0044 \mathrm{Jg}^{-1}$ respectively (ctu - computer time unit). A $12 \mathrm{GPa}$ upper limit for bone stiffness, $\rho_{c b}$, was used [20, 21]. The adaptation of the surrounding trabecular bone was assumed to be negligible, as resorption of existing bone stock due to implant stress shielding is observed only in the long term [3].

An established density-modulus relationship from literature $\left(E=3790 \rho^{3}\right)$ was used to update the stiffness matrix for the next increment [22]. The solver time step was set to $0.1 \mathrm{ctu}$, until the rate of change of the number of remodeling elements reaches zero. Thereafter, for computational efficiency, adaptive time stepping at 1.2x of the previous step was used until the FEA reaches equilibrium $\left(\mathrm{d} \rho_{\text {average }}<0.005 \%\right.$ ), as the rate of change slows asymptotically towards equilibrium. The time units used should be considered arbitrary, as the time correspondence for sheep has not been established in literature and histology results were only available at one time point in this study. Quantification of bone area ratio followed the same procedure as histological analysis.

\subsection{Failure risk assessment}

The locations of maximum von Mises stress were identified, and path plots drawn along the loading direction of the maximum von Mises stress to evaluate the stress distribution across the implants. The magnitude of the maximum stress was compared against the strength and fatigue limits of titanium alloys, as listed in Table 1.

Table 1: Mechanical properties of additively manufactured titanium and titanium-tantalum alloys. Fatigue strength at $10^{7}$ cycles $[5,18,23,24]$

\begin{tabular}{|lll|}
\hline & Ti6Al4V & TiTa \\
\hline Yield strength & $810 \mathrm{MPa}$ & $375 \mathrm{MPa}$ \\
\hline Tensile Strength & $860 \mathrm{MPa}$ & $600 \mathrm{MPa}$ \\
\hline Fatigue Strength & $350 \mathrm{MPa}$ & $400 \mathrm{MPa}$ \\
\hline
\end{tabular}

A

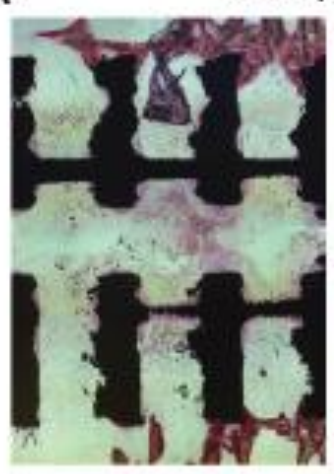

$1500 \mu \mathrm{m}$ implant

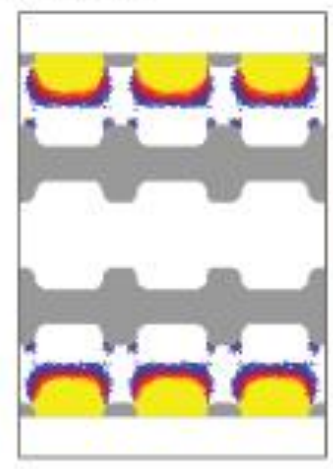

B

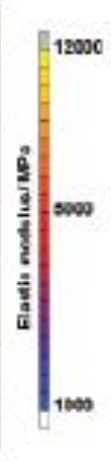

$700 \mu \mathrm{m}$ implant

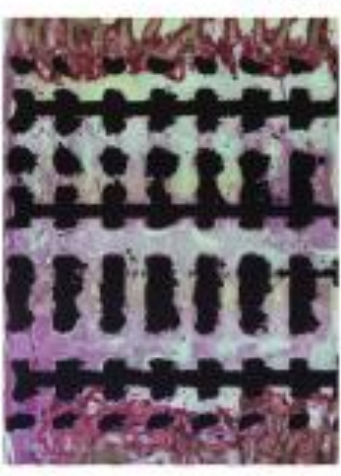

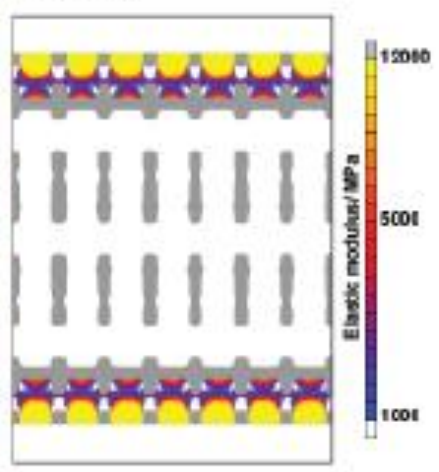

Fig. 3: Verification of bone formation between histology results and FEA predictions for (A) $1500 \mu \mathrm{m}$ implant and (B) $700 \mu \mathrm{m}$ implant. Pink regions in histology slides indicate bone formation. 


\section{RESULTS AND DISCUSSION}

The regions of bone formation obtained experimentally, as shown in the histological results, are compared against the FEA predictions at equilibrium in Fig. 3. The FEA results showed good correlation with the histology results in predicting bone formation that is limited to the outer pores for both implants. The BIC (bone-to-implant contact) measured from the histology results was $9.8 \pm 5.0 \%$ and $10.7 \pm 4.9 \%$ for the $1500 \mu \mathrm{m}$ and $700 \mu \mathrm{m}$ implant respectively, which was significantly different $(\mathrm{p}=0.057)$. FEA over-predicted the BIC, which was $19.8 \pm 11.2 \%$ and $22.6 \pm 6.4 \%$ for the 1500 $\mu \mathrm{m}$ and $700 \mu \mathrm{m}$ implants, respectively. The FEA results predicted a density gradient remaining in the $1500 \mu \mathrm{m}$ implant at equilibrium, whereas fully dense bone was predicted to form in the outer pores, with the struts blocking bone formation on its underside. However, overall bone formation filled almost the entire second layer of pores in the $700 \mu \mathrm{m}$ implant, whereas bone formation was minimal and limited to the region near the geometric shape change in the $1500 \mu \mathrm{m}$ implant.

The stress patterns in the porous implants before and after bone remodeling showed that there is a shift in the location of highest stress concentration towards the interior of the implant (Fig. 4). Before remodeling, the highest stress was located at the sharp angle at the partial holes, followed by the narrowing of the plate due to the holes, and the strut fillets. Partial bone formation into the second layer of pores reduced the stress concentration at the partial holes for both implants. There was a slight reduction in stress along the holes in the second layer of the pores for the $700 \mu \mathrm{m}$ implants, due to the higher volume of bone ingrowth predicted. However, in the $1500 \mu \mathrm{m}$ implants, there was an increase in stress along the holes in the second layer of the pores, where bone formation had not occurred. Before remodeling, the peak von Mises stress was above the fatigue limit at $10^{7}$ cycles for additive manufactured Ti6Al4V for both implants. Remodeling reduced the stress concentration of the $1500 \mu \mathrm{m}$ implant to below the fatigue limit, but the stress concentration was still higher than the safety limit for the $700 \mu \mathrm{m}$ implant. The highest stress concentrations reduced in both implants, by $21 \%$ from $383 \mathrm{MPa}$ to $301 \mathrm{MPa}$ for the $1500 \mu \mathrm{m}$ implant, and 23\% from $534 \mathrm{MPa}$ to $411 \mathrm{MPa}$ for the $700 \mu \mathrm{m}$ implant (Table 2). The higher stresses in the $700 \mu \mathrm{m}$ implant were due to its higher porosity (75\%) and use of thinner strut and plates, compared to the $1500 \mu \mathrm{m}$ implant $(53 \%)$.

A $\quad 1500 \mu \mathrm{m}$ implant
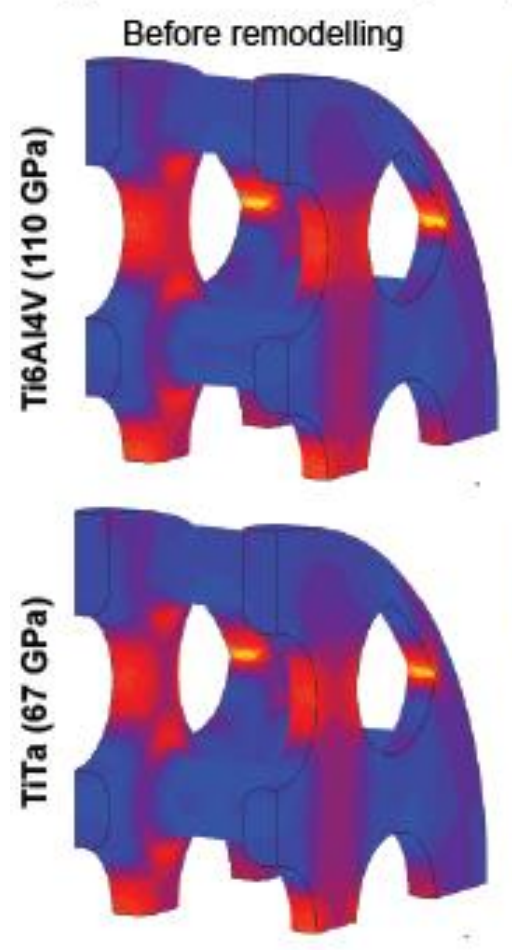

After remodelling
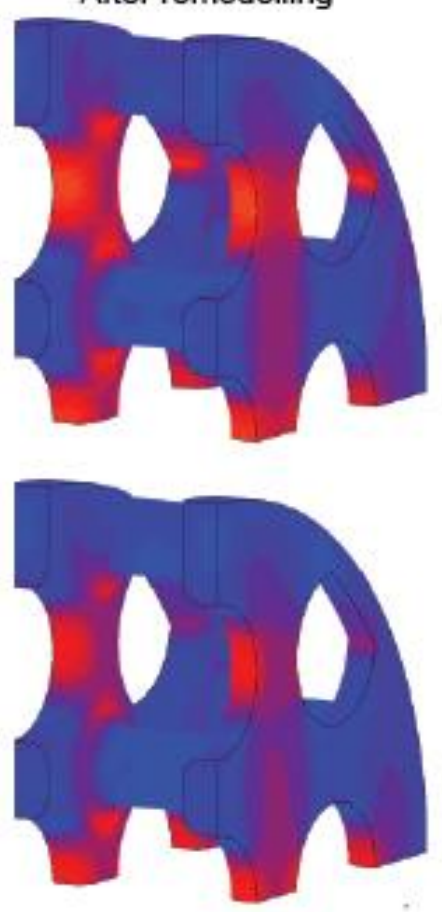

B
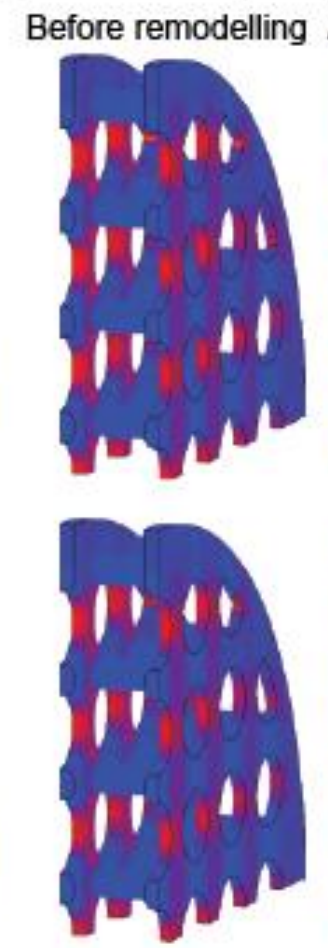

$700 \mu \mathrm{m}$ implant
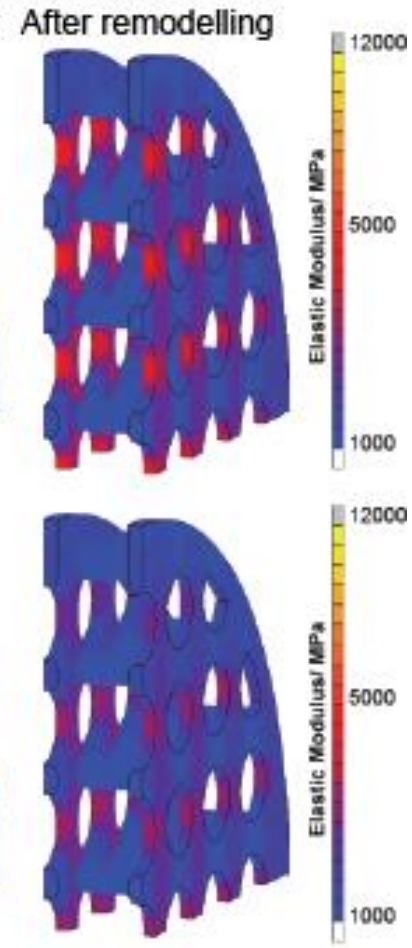

Fig. 4: Effect of bone formation on stress distribution in the implant by changing the material properties.

(A) $1500 \mu \mathrm{m}$ implant. (B) $700 \mu \mathrm{m}$ implant. 

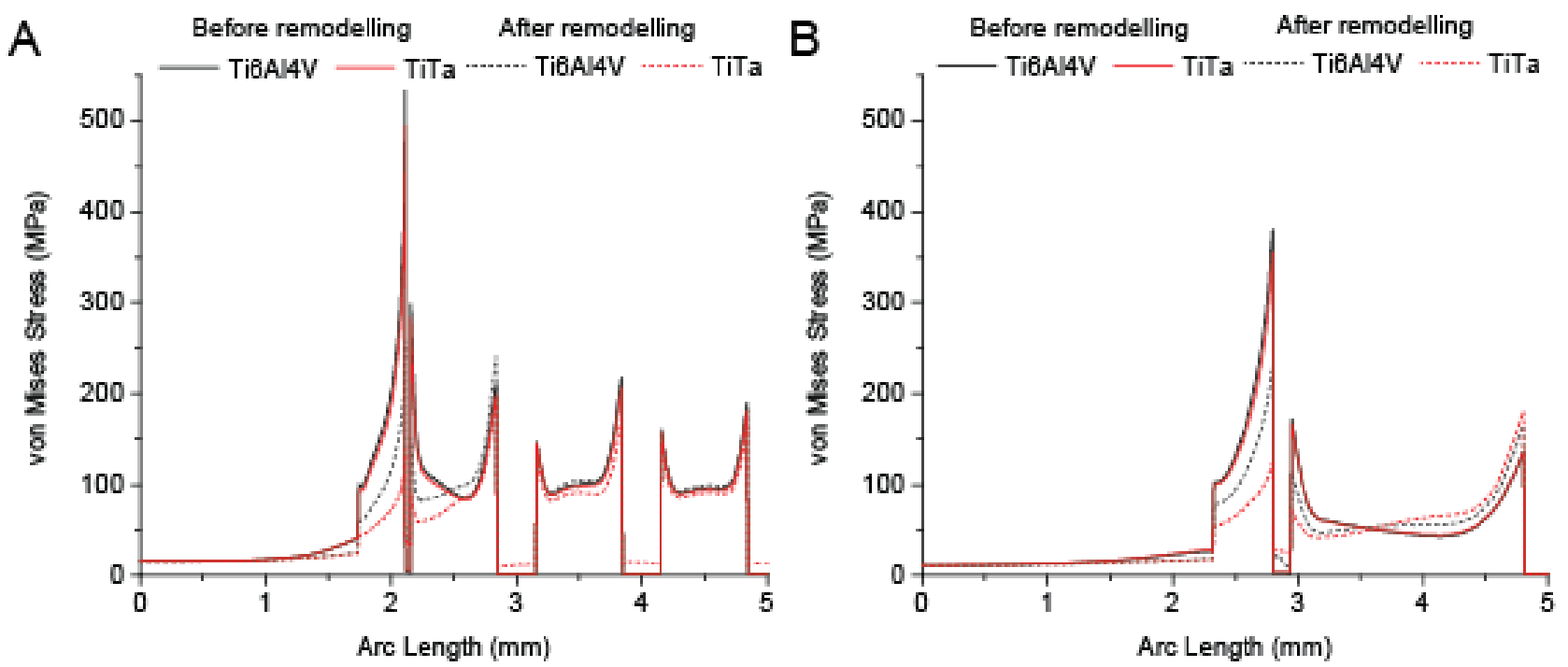

Fig. 5: Path plots drawn in loading direction, through edge of most proximal partial implant hole (initial maximum nodal stress).

(A) $1500 \mu \mathrm{m}$ implant; (B) $700 \mu \mathrm{m}$ implant. Black and red lines indicate results for Ti6Al4V (110 GPa) and TiTa (67 GPa).

Path plots drawn along the loading direction of the initial maximum von Mises stress showed that the stress levels along the plate reduced up to the level of bone ingrowth into the implant, while the stresses increased in the deeper region of the same layer (Fig. 5). The von Mises stress at the partial holes reduced from $383 \mathrm{MPa}$ to $232 \mathrm{MPa}$ for the $1500 \mu \mathrm{m}$ implant, and from $534 \mathrm{MPa}$ to $259 \mathrm{MPa}$ in the $700 \mu \mathrm{m}$ implant, below the fatigue failure strength of additively manufactured Ti6Al4V, as a result of bone formation in that region. However, partial bone formation to the second layer caused the stresses along the plates in the second layer to increase from 282 to $301 \mathrm{MPa}$, and 398 to $411 \mathrm{MPa}$ in the $1500 \mu \mathrm{m}$ and $700 \mu \mathrm{m}$ implant, respectively. The stress levels in the deeper layers were unaffected by the process of bone formation.

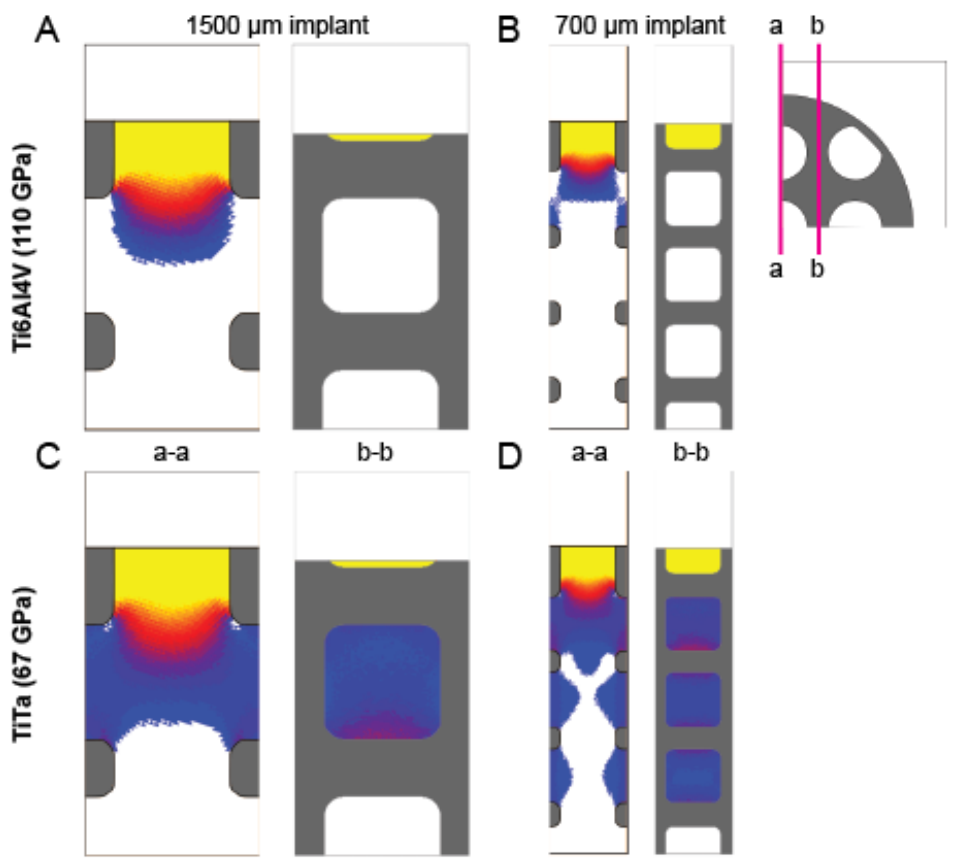

Fig. 6: Extent of bone formation at equilibrium at two different regions through the implant. (A-B) Ti6Al4V, 110GPa. (C-D) TiTa, 67GPa. 
For the TiTa implant (67 GPa), bone formation filled the first layer in both the $700 \mu \mathrm{m}$ and $1500 \mu \mathrm{m}$ implant (Fig. 6). The second layer was mostly filled with bone in the $1500 \mu \mathrm{m}$ implant, with a density gradient from the exterior to the interior. In the $700 \mu \mathrm{m}$ implant, bone formation with elastic moduli ranging from 1-12 GPa covered the holes along the plates. There was minimal bone formation immediately beneath the struts, but most of the regions below the struts were largely unfilled. Stress reduction in the TiTa implant was larger than for Ti6Al4V, especially at the partial holes and the holes along the plates (Fig. 4). At the partial holes, the peak stress reduced from $495 \mathrm{MPa}$ to $139 \mathrm{MPa}$ for the $700 \mu \mathrm{m}$ implant, and from $356 \mathrm{MPa}$ to $124 \mathrm{MPa}$ for the $1500 \mu \mathrm{m}$ implant (Fig. 5). However, the highest stress concentration at equilibrium was $281 \mathrm{MPa}$ and $262 \mathrm{MPa}$ for the $700 \mu \mathrm{m}$ and $1500 \mu \mathrm{m}$ implant respectively. The reduction in stress concentrations is consistent with previous studies that modelled different stages of bone growth in solid and porous implant and showed that higher bone formation protects the implant more effectively from fracture $[6,15]$.

\section{CONCLUSIONS}

The effect of partial bone formation into the pores of additively manufactured implants with two different porosities was evaluated. A mechanoregulation algorithm was applied to predict bone remodeling, and the results were verified against histology results from retrieved implants after a 6 weeks follow-up. The FEA models were able to predict the trend in bone formation, but over-predicted the extent of bone-to-implant contact. Both the in-vivo and FEA models showed limited amount of bone ingrowth, which was found to be due to stress shielding caused by the stiff outer strut. Prediction of bone ingrowth was thus higher in the $1500 \mu \mathrm{m}$ implant as the outermost strut was located further into the implant. The results showed that bone remodeling reduces the maximum von Mises stress of Ti6Al4V implants by more than 20\%, but the maximum implant stress is still not within safety limits of additively manufactured Ti6Al4V. Bone remodeling also shifted the location of the highest stress concentration from the most proximal partial holes to the first layer of the struts. FEA simulations for TiTa implant material (lower Young's modulus) predicted significantly increased bone ingrowth, although the elastic modulus of additional bone formed was closer to that of trabecular bone than cortical bone. Nevertheless, the higher amount of bone formed reduced stress concentrations by $26 \%$ and $43 \%$ respectively for the $1500 \mu \mathrm{m}$ and $700 \mu \mathrm{m}$ implants. Compared to Ti6Al4V, the highest von Mises stress at equilibrium of an implant additively manufactured by TiTa is within safe limits of the material. The results from this study show that the design and use of extensively porous implants in load bearing situations require additional safety checks through FEA simulations of bone remodeling, as bone ingrowth changes the stress distribution within the implant. The level of bone ingrowth also determines the extent to which it reinforces the implant, thus protecting it from failure. Partial bone formation in an implant designed to achieve complete ossoeintegation may put the implant at risk of fatigue failure. The use of TiTa has demonstrated significant effects in reducing stress concentrations and should be considered for implant designs.

\section{REFERENCES}

[1] Balla, V. K., Bodhak, S., Bose, S. and Bandyopadhyay, A., "Porous tantalum structures for bone implants: fabrication, mechanical and in vitro biological properties," Acta Biomater. 6, 3349-3359 (2010).

[2] Biemond, J. E., Hannink, G., Verdonschot, N. and Buma, P., "Bone ingrowth potential of electron beam and selective laser melting produced trabecular-like implant surfaces with and without a biomimetic coating," J. Mater. Sci. Mater. Med. 24, 745-753 (2013).

[3] Coathup, M. J., Batta, V., Pollock, R. C., Aston, W. J., Cannon, S. R., Skinner, J. A., Briggs, T. W., Unwin, P. S. and Blunn, G. W., "Long-term survival of cemented distal femoral endoprostheses with a hydroxyapatite-coated collar: a histological study and a radiographic follow-up,” J. Bone Joint Surg. Am. 95, 1569-1575 (2013).

[4] Cheong, V. S., Fromme, P., Mumith, A., Coathup, M. and Blunn, G., "Novel adaptive finite element algorithms to predict bone ingrowth in additive manufactured porous implants " J. Mech. Behav. Biomed. Mater. 87, 230-239 (2018).

[5] Ghouse, S., Babu, S., Nai, K., Hooper, P. A. and Jeffers, J. R. T., "The influence of laser parameters, scanning strategies and material on the fatigue strength of a stochastic porous structure," Addit. Manuf. 22, 290-301 (2018).

[6] Cheong, V. S., Fromme, P., Coathup, M. J., Mumith, A. and Blunn, G. W., "Partial Bone Formation in Additive Manufactured Porous Implants Reduces Predicted Stress and Danger of Fatigue Failure," Ann. Biomed. Eng. (2020).

[7] Mumith, A., Coathup, M., Chimutengwende-Gordon, M., Aston, W., Briggs, T. and Blunn, G., "Augmenting the osseointegration of endoprostheses using laser-sintered porous collars: an in vivo study," Bone \& Joint J. 99-B, 276282 (2017). 
[8] de Wild, M., Zimmermann, S., Rüegg, J., Schumacher, R., Fleischmann, T., Ghayor, C. and Weber, F. E., "Influence of Microarchitecture on Osteoconduction and Mechanics of Porous Titanium Scaffolds Generated by Selective Laser Melting," 3D Print. Addit. Manuf. 3, 142-151 (2016).

[9] Mumith, A., Blunn, G., Cheong, V. S., Fromme, P. and Coathup, M., "The effect of strontium and silicon substituted hydroxyapatite electrochemical coatings on bone ingrowth and osseointegration of selective laser sintered porous metal implants," PLoS One 15, e0227232 (2020).

[10] Reznikov, N., Boughton, O. R., Ghouse, S., Weston, A. E., Collinson, L., Blunn, G. W., Jeffers, J. R. T., Cobb, J. P. and Stevens, M. M., "Individual response variations in scaffold-guided bone regeneration are determined by independent strain- and injury-induced mechanisms," Biomaterials 194, 183-194 (2019).

[11] U.S. Food and Drug Administration, "Class 2 Device Recall Tritanium Posterior Lumbar (PL) cage". 13 December 2018, < https://www.accessdata.fda.gov/scripts/cdrh/cfdocs/cfRES/res.cfm?id=169021>(24 March 2020).

[12] Carli, A. V., Warth, L. C., de Mesy Bentley, K. L. and Nestor, B. J., "Short to midterm follow-up of the tritanium primary acetabular component: a cause for concern," J. Arthroplasty 32, 463-469 (2017).

[13] Hedayati, R., Janbaz, S., Sadighi, M., Mohammadi-Aghdam, M. and Zadpoor, A. A., "How does tissue regeneration influence the mechanical behavior of additively manufactured porous biomaterials?," J. Mech. Behav. Biomed. Mater. 65, 831-841 (2017).

[14] Cheong, V. S., Blunn, G., Coathup, M. and Fromme, P., “Adaptive 3D Finite Element Analysis Model to Simulate Extracortical Bone Growth,” Comput. Methods Biomech. Biomed. Eng. 21, 129-138 (2018).

[15] Fromme, P., Blunn, G. W., Aston, W. J., Abdoola, T., Koris, J. and Coathup, M. J., “The Effect of Bone Growth onto Massive Prostheses Collars in Protecting the Implant from Fracture," Med. Eng. Phys. 41, 19-25 (2017).

[16] Taylor, W. R., Ehrig, R. M., Heller, M. O., Schell, H., Seebeck, P. and Duda, G. N., "Tibio-femoral joint contact forces in sheep," J. Biomech. 39, 791-798 (2006).

[17] Huiskes, R., "If bone is the answer, then what is the question?," J. Anat. 197, 145-156 (2000).

[18] Zhou, Y. L., Niinomi, M. and Akahori, T., "Effects of Ta content on Young's modulus and tensile properties of binary Ti-Ta alloys for biomedical applications," Mater. Sci. Eng. A 371, 283-290 (2004).

[19] Frost, H. M., "Bone's mechanostat: a 2003 update," Anat. Rec. Part A 275, 1081-1101 (2003).

[20] Tomaszewski, P. K., Verdonschot, N., Bulstra, S. K. and Verkerke, G. J., "A comparative finite-element analysis of bone failure and load transfer of osseointegrated prostheses fixations," Ann. Biomed. Eng. 38, 2418-2427 (2010).

[21] Dickinson, A., Taylor, A. and Browne, M., "Implant-bone interface healing and adaptation in resurfacing hip replacement," Comput. Methods Biomech. Biomed. Engin. 15, 935-947 (2012).

[22] Carter, D. and Hayes, W., "The compressive behavior of bone as a two-phase porous structure," J. Bone Joint Surg. Am. 59, 954-962 (1977).

[23] Wycisk, E., Siddique, S., Herzog, D., Walther, F. and Emmelmann, C., "Fatigue Performance of Laser Additive Manufactured Ti-6Al-4V in Very High Cycle Fatigue Regime up to 109 Cycles," Front. Mater. 2, 72 (2015).

[24] Brandl, E., Leyens, C. and Palm, F., "Mechanical Properties of Additive Manufactured Ti-6Al-4V Using Wire and Powder Based Processes,” IOP Conf. Ser.: Mater. Sci. Eng. 26, 012004 (2011). 